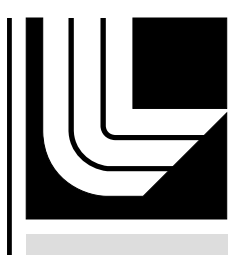

LAW RENCE LIVERMORE N A TION A L LABORATORY

Ion Drifts in a Snowflake Divertor

D. D. Ryutov, M. V. Umansky

October 16, 2009

Physics of Plasmas 
This document was prepared as an account of work sponsored by an agency of the United States government. Neither the United States government nor Lawrence Livermore National Security, LLC, nor any of their employees makes any warranty, expressed or implied, or assumes any legal liability or responsibility for the accuracy, completeness, or usefulness of any information, apparatus, product, or process disclosed, or represents that its use would not infringe privately owned rights. Reference herein to any specific commercial product, process, or service by trade name, trademark, manufacturer, or otherwise does not necessarily constitute or imply its endorsement, recommendation, or favoring by the United States government or Lawrence Livermore National Security, LLC. The views and opinions of authors expressed herein do not necessarily state or reflect those of the United States government or Lawrence Livermore National Security, LLC, and shall not be used for advertising or product endorsement purposes. 


\title{
Ion drifts in a snowflake divertor
}

\author{
D.D. Ryutov, M.V. Umansky \\ Lawrence Livermore National Laboratory, Livermore, CA 94551, USA
}

\begin{abstract}
Prompt losses of ions having turning points in the vicinity of the poloidal field null of a snowflake divertor are analyzed. Classification of the ion trajectories is presented. It is concluded that prompt losses in a snowflake affect a broader zone than in the standard X-point divertor. The size of the phase-space "hole" produced by prompt losses is evaluated.

PACS Numbers: 52.20.Dq; 52.55.Rk

We consider ion drifts in the vicinity of the null-point of the snowflake divertor [1] leading to the prompt losses of these ions. In the case of the standard X-point geometry the prompt losses have been studied in a number of publications (e.g., [2-4] and references therein), whereas the snowflake geometry has not been explored in this context.

Consider particles having initial positions on the closed flux surfaces in the vicinity of the separatrix. Some of these particles have turning points near the poloidal magnetic field null and, due to the weakness of the poloidal magnetic field there, spend a long time in the turn-around area. As noted in Ref. [4], the drift caused by the radial variation of the toroidal field will have a strong effect on the trajectories of such particles. In particular, for a "normal" direction of the drift (from inside to outside of the separatrix) it would cause prompt losses of a certain group of ions. Although this process is relatively insignificant in terms of the total power and particle balance, it may be important as a source of non-ambipolar ion losses and, therefore, may lead to generation
\end{abstract}


of the radial electric field inside the separatrix [3,4]. It may affect the behavior of partially-ionized impurities in the divertor and may also be of some diagnostic value.

In a snowflake divertor, the poloidal magnetic field near the null point scales as a square of the distance from the null, not linearly as in a "standard" X-point divertor. This, obviously, leads to a larger role of the aforementioned effects on the plasma behavior near the null and serves as a motivation for the present study.

In this brief communication we are going to present a general drift trajectory analysis and evaluate prompt losses in the simplest case where both the effects of a radial electric field and particle collisions can be neglected. This can be considered as a first step to a more detailed assessment of prompt ion losses in a snowflake divertor.

The geometry of the system is illustrated in Fig. 1. The poloidal field null serves as the origin for the Cartesian coordinates $(x, z)$ in the poloidal plane; the major radius of the null-point is $R$. The poloidal magnetic field can be characterized by the magnetic flux function $\Psi(x, z)$; the components of the poloidal field are: $B_{x}=-(1 / r) \partial \Psi / \partial z$, $B_{z}=(1 / r) \partial \Psi / \partial x$, where $r$ is the major radius of the observation point, $r=R+x$. The null of the flux function $\Psi(x, z)$ is chosen at the separatrix; the flux function is negative inside and positive outside the separatrix. Throughout this paper, we assume that the toroidal magnetic field is stronger than the poloidal field and, therefore, $B=\sqrt{B_{T}^{2}+B_{P}^{2}} \approx B_{T} \propto 1 / r$.

The parallel ion velocity is determined by the conservation of the ion energy $W$ and magnetic moment. We consider the ions moving near the separatrix and are interested in the ions whose parallel motion has a turning point not far from the nullpoint. Denoting the $x$-coordinate of the turning point by $x^{*}$, one can write the following expression for the parallel velocity in terms of the particle energy $W$ : 
$\mathrm{v}_{\mathrm{\|}}=\mp \sqrt{\frac{2 W}{m}} \sqrt{\frac{x-x^{*}}{R}}$,

where we assume that $x^{*}<<R$. The sign "minus" ("plus") corresponds to the particle moving to the left (to the right). We assume that the ion gyroradius is small compared to other spatial scales and use zero gyro-radius drift approximation, leaving accounting for the next-order effects in the ion gyroradius [5] for the future work.

The ion drift is determined by the curvature and gradB drifts (in this paper we neglect the possible presence of the electric field). The analysis is significantly simplified by the fact that the ion parallel velocity not far from the turning point is much smaller than the ion perpendicular velocity. This allows us to neglect the curvature drift compared to the gradB drift, thereby yielding very simple equations of motion:

$$
\dot{x}=-\frac{\mathrm{v}_{\|}}{B_{T} r} \frac{\partial \Psi}{\partial z} ; \dot{z}=\frac{\mathrm{v}_{\|}}{B_{T} r} \frac{\partial \Psi}{\partial x}-\frac{c}{e} \frac{W}{r B_{T}} .
$$

Substituting Eq. (1) into Eqs (2), one can easily find the equation for the particle drift trajectory:

$$
\Psi \pm \frac{c \sqrt{2 m W}}{e} \sqrt{\frac{x-x^{*}}{R}}=\Psi_{0}+\frac{c \sqrt{2 m W}}{e} \sqrt{\frac{x_{0}-x^{*}}{R}}
$$

where the upper (lower) sign corresponds to the particle moving to the left (to the right) in the geometry of Fig. 1, and the constants $x_{0}$ and $\Psi_{0}$ define the initial position of the particle. To be specific, we assume that initially the particle is situated to the right of the null point and moves to the left (this determines the sign of the last term in the right-hand side). The other possible cases can be treated similarly. One can relate $\Psi_{0}$ to the distance $\Delta_{0}$ between the flux surface and the separatrix in the midplane: one has 
$\Psi_{0}=-B_{P M}(R+a) \Delta_{0}$, where $B_{P M}$ is the poloidal magnetic field on the separatrix at the midplane and $a$ is the minor radius.

We use the same model of the snowflake configuration as the one described in Refs. $[1,4,5]$, with the branches of the separatix oriented as shown in Fig. 1. In this case, the flux function in the divertor area can be represented as [4]:

$$
\Psi=\eta \frac{B_{P M} R}{a^{2}}\left(x^{2} z-\frac{z^{3}}{3}\right),
$$

where $\eta$ is a numerical coefficient of order 1 depending on the details of the geometry. In the model considered in Ref. 4, it was equal to 1.5. It is sometimes more convenient to characterize the flux surface not by the parameter $\Psi_{0}$ (or $\left.\Delta_{0}\right)$, but by its shortest distance $z_{\min }$ to the magnetic field null (Fig. 1). For the flux function (4), one has $\Psi_{0}=-\eta \frac{B_{P M}}{a^{2}} \frac{z_{\min }^{3}}{3}$

For the snowflake flux function (4), one can be rewrite Eq. (3) in terms of dimensionless coordinates $\xi$ and $\zeta$ related to $x$ and $z$ by $x=a \varepsilon^{2 / 5} \xi ; z=a \varepsilon^{2 / 5} \xi$, with

$$
\varepsilon=\frac{c \sqrt{2 m W}}{\eta a B_{P M} e} \sqrt{\frac{a}{R}} .
$$

The parameter $\varepsilon$ is small, on the order of the ion poloidal gyro-radius over the minor radius. For a tokamak of DIII-D scale it is $\sim 0.05$. In these variables, the trajectory equation acquires a universal form

$$
\xi^{2} \zeta-\frac{\zeta^{3}}{3} \pm \sqrt{\xi-\xi^{*}}=-\frac{\xi_{\min }^{3}}{3}+\sqrt{\xi_{0}-\xi^{*}}
$$

A few solutions of this equation are illustrated in Fig. 2 for $\xi *=-1$ and trajectories passing through the point $\xi=\xi_{0}=0, \zeta=\zeta_{0}$ (note that, for $\xi_{0}=0$, one has $\zeta_{\min }=\zeta_{0}$ ). For large-enough 
$\zeta_{0}$, one has a trajectory 1 that is similar to a standard banana orbit, for which the particles stay inside the separatrix. For small-enough $\zeta_{0}$, the particle is promptly lost (trajectory 2). The marginal $\zeta_{0}$ corresponds to a singular trajectory 3 (which contains a selfintersection).

The singular point corresponds to a situation where derivatives of the 1.h.s. of Eq. (6) over $\xi$ and $\xi$ are zero. Imposing this condition, one finds the following parametric equation which determines the marginal $\xi_{\min }$ for any choice of $\xi_{*}$ and $\xi_{0}>\xi_{*}$ :

$$
\frac{\xi_{\min }^{3}}{3}=\frac{1}{4 t^{2}}-\frac{2 t^{3}}{3} \pm \sqrt{\xi_{0}+\frac{1}{16 t^{4}}-t} ;-\xi^{*}=\frac{1}{16 t^{4}}-t,
$$

where $t$ is a parameter. The sign "minus" here corresponds to the particles that are initially moving to the left. The plot of $\zeta_{\min } \mathrm{vs}-\xi *$ for several values of $\xi_{0}$ is presented in Fig. 3, with the upper branch corresponding to the particles that are moving to the left (high-field side) in the starting point, and the lower branch corresponds to the particles moving in the opposite direction.

Eq. (7) shows, that, for particles starting in the vicinity of the null, $\xi_{0} \sim 1$, and having a significant parallel energy, so that $-\xi^{*}>>1$, there is a simple approximate relation between $\xi_{\min }$ and $-\xi^{*}$ independent on $\xi_{0}$ :

$$
\frac{\zeta_{\min }^{3}}{3} \approx 2 \sqrt{-\xi^{*}} \text { (upper branch); } \quad \frac{\zeta_{\min }^{3}}{3} \approx \frac{2^{1 / 6} 3^{1 / 2}}{\sqrt{-\xi^{*}}} \text { (lower branch) }
$$

The particles moving initially to the left (right) are lost if $\zeta_{0}$ lies below the upper (lower) branch, $\zeta_{\min }=\zeta_{\min }^{-}\left(-\xi^{*}\right)\left(\zeta_{\min }=\zeta_{\min }^{+}\left(-\xi^{*}\right)\right)$, where the sign "minus" corresponds to the upper branch. We note that the loss region, for a given $\zeta_{0}$, extends far into domain of large $-\xi^{*}$; in other words, particles with the turning points far into the inboard 
direction are lost. However, when $-\xi^{*}$ becomes too large, the particles become transit particles circulating around the magnetic axis and not experiencing prompt losses. Therefore, there is an upper bound on $-\xi^{*}$, beyond which the prompt loss is turned off. This upper bound approximately corresponds to $-x^{*} \approx-a$, or

$$
-\xi^{*}=-\xi^{*}{ }_{c r i t} \approx(a / R) \varepsilon^{-2 / 5}
$$

(a vertical line in Fig. 3, for $a \approx 0.4 \mathrm{R}, \varepsilon=0.05$ ).

To illustrate the application of these results, consider fraction $f$ of particles promptly lost from an initially isotropic distribution with the same initial energy $W$. We look for the dependence of this fraction on the distance $z$ from the origin, along the vertical axis (i.e., we take $\xi_{0}=0$ ). For a given $\xi_{0}$, lost are the particles with a small-enough value of $\left|v_{\|}\right|$. Those occupy a strip of the surface area of $2 \pi v\left(v_{\| /}^{+}-v_{\|}^{-}\right)$on the surface of a velocity-space sphere with the area $4 \pi \mathrm{v}^{2}$; here the signs “-“" (“+”) correspond to particles moving to the left (right) at the initial point. Noting that, for $\xi_{0}=0$, $\mathrm{v}_{\| /}^{ \pm}= \pm \varepsilon^{1 / 5} \mathrm{v}_{\perp} \sqrt{a / R} \sqrt{-\xi *}$ and using Eq. (7), one finds

$$
f\left(\zeta_{0}\right)= \begin{cases}a / R, & \zeta_{0}<\xi_{\min }^{+}\left(-\xi_{c r i t}^{*}\right) \\ (a / 2 R)+\frac{\varepsilon^{1 / 5}}{2} \sqrt{\frac{a}{R}} \sqrt{-\xi^{*+}}, & \zeta_{\min }^{+}\left(-\xi_{c r i t}^{*}\right)<\zeta_{0}<\zeta_{\min }^{+}(0) \\ (a / 2 R)-\frac{\varepsilon^{1 / 5}}{2} \sqrt{\frac{a}{R}} \sqrt{-\xi^{*-}}, & \zeta_{\min }^{-}(0)<\zeta_{0}<\zeta_{\min }^{-}\left(-\xi_{c r i t}^{*}\right) \\ 0, & \zeta_{0}>\zeta_{\min }^{-}\left(-\xi_{c r i t}^{*}\right),\end{cases}
$$

where $\xi^{* \pm}$ is a solution of equation $\xi_{0}=\zeta_{\text {min }}^{ \pm}\left(-\xi^{*}\right)$ and $\xi_{c r i t}^{*}$ is defined by Eq. (9). The plot of the function $f$ is presented in Fig. 4 , for $a / R=0.4$ and $\varepsilon=0.05$. As a characteristic width (in $z$, or, equivalently, in $\zeta$ ) of the zone from which the prompt loss occurs, one can take 
the tip of the curves shown in Fig. 3, i.e., the point $\zeta_{w}=\zeta_{\min }^{ \pm}(0)$, with the subscript $w$ referring to the width. Eq. (7) shows, that for $\xi_{0}=0$ this point is $\zeta_{w}=5^{1 / 3} 2^{-7 / 15} \approx 1.2$, or

$$
z_{w}=1.2 a \varepsilon^{2 / 5}
$$

Consider for comparison the standard X-point divertor, for which the flux function is

$$
\Psi=\eta_{X} B_{p m} R \frac{x^{2}-z^{2}}{a^{2}}
$$

where $\eta_{X}$ is a numerical factor of order 1 accounting for the details of the geometry.

Introducing dimensionless variables $\xi$ and $\zeta$ according to $x=\varepsilon^{2 / 3} a \xi, z=\varepsilon^{2 / 3} a \zeta$ and using Eqs. (3) and (11), one finds the following trajectory equations:

$$
\xi^{2}-\zeta^{2} \pm \sqrt{\xi-\xi^{*}}=-\zeta_{0}^{2} \pm \sqrt{\xi_{0}-\xi^{*}}
$$

A set of trajectories is shown in Fig. 5 for $\xi_{0}=0, \xi_{0}=1$ and several values of $\xi *$. These results are consistent with those presented in a detailed paper [6].

An analog of Eq. (7) reads now as

$$
-\xi^{*}=\frac{1}{16 t^{2}}-t ; \quad \zeta_{\min }^{ \pm}=\left[\frac{1}{4 t}-t^{2} \pm \sqrt{\xi_{0}+\frac{1}{16 t^{2}}-t}\right]^{1 / 2}
$$

The plot of $\xi_{\min }^{ \pm}\left(-\xi^{*}\right)$ for $\xi_{0}=0$ is shown in Fig. 3 by dashed line. An analog of the equation (11) reads now as

$$
z_{w}=0.687 a \varepsilon^{2 / 3}
$$

In other words, the width of the zone strongly affected by prompt losses is by a factor of $\left(1 / \varepsilon^{4 / 15}\right)$ broader in the snowflake divertor than in an X-point divertor. Numerically, this is a factor between 2 to 3 for most of the existing tokamaks. 
The characteristic time $\tau_{\text {loss }}$ for the ion to leave the zone of the prompt losses can be evaluated by dividing the distance $z_{\mathrm{w}}$ (Eq. (11)) by the drift velocity, $c W / e R B_{T}$. This yields the following estimate of the loss time:

$$
\tau_{\text {loss }} \sim \frac{R}{\mathrm{v}_{T i}}\left(\frac{a}{\rho_{i} *}\right)^{3 / 5} \frac{B_{T}}{B_{P}}\left(\frac{a}{R}\right)^{1 / 5},
$$

where $\rho_{i} *$ is the poloidal ion gyroradius at the midplane, and $\mathrm{B}_{\mathrm{P}}$ is the poloidal magnetic field strength in the midplane. Collisions do not affect this loss channel provided the ion scattering time over the angle $\sim$ the angular width of the loss-strip (see discussion before Eq. (10)), which is $\tau_{\text {scatt }} \sim \tau_{i i}(a / R)$, with $\tau_{i i}$ being the standard ion collision time. The ion temperature and density here correspond to the zone at the distance of order of $z_{\mathrm{W}}$ above the null-point. Taking as an example $T_{i} \sim 300 \mathrm{eV}, n \sim 3 \times 10^{13} \mathrm{~cm}^{-3}, a / \rho_{i}^{*} \sim 20, a / R=0.4$, $B_{T} / B_{P} \sim 5, R \sim 1.5 \mathrm{~m}$, one finds that $\tau_{\text {loss }} / \tau_{\text {scatt }} \sim 0.3$; in other words, the scattering does not play a dominant role. The result, however, strongly depends on the ion temperature and may vary significantly from one device to another.

In summary: the mechanism of prompt ion losses considered in Refs. $[2,3,6]$ for the $\mathrm{X}$-point divertor is analyzed for the snowflake configuration. The general classification of the ion trajectories in the vicinity of the null-point is presented. The width of the affected zone and the loss time is evaluated. It is concluded that this mechanism affects a broader zone than in the standard X-point divertor.

The authors are grateful to I. Joseph, T. Rognlien and X. Xu for helpful comments. Prepared by LLNL under Contract DE-AC52-07NA27344. 


\section{References}

1. D.D. Ryutov. 'Geometrical Properties of a "Snowflake" Divertor.' Phys. Plasmas, 14, 064502 (2007).

2. K.C. Shaing et al. Phys. Fluids, B2, 1492 (1990).

3. A.V. Chankin, G.M. McCracken. "Loss ion orbits at the tokamak edge." Nuclear Fusion, 33, 1459 (1993)

4. C.S. Chang, S. Kue, H. Weitzner. "X-transport: A baseline nonambipolar transport in a diverted tokamak plasma edge.” Phys. Plasmas, 9, 3854 (2002).

5. H. Weitzner, C.S. Chang. "Single particle motion near an X point and separatrix." Phys. Plasmas, 11, 3060 (2004).

6. D.D. Ryutov, R.H. Cohen, T.D. Rognlien, M.V. Umansky. "Magnetic field structure of a snowflake divertor.” Phys. Plasmas, 15, 092501 (2008).

7. M.V. Umansky, R.H. Bulmer, R.H. Cohen, T.D. Rognlien. D.D. Ryutov. “Analysis of geometric variations in high-power tokamak divertors." Nuclear Fusion, 49, 075005 (2009).

8. S. Ku, H. Baek, C.S. Chang. "Property of an X-point generated velocity-space hole in a diverted tokamak plasma edge." Phys. Plasmas, 11, 5626 (2004). 


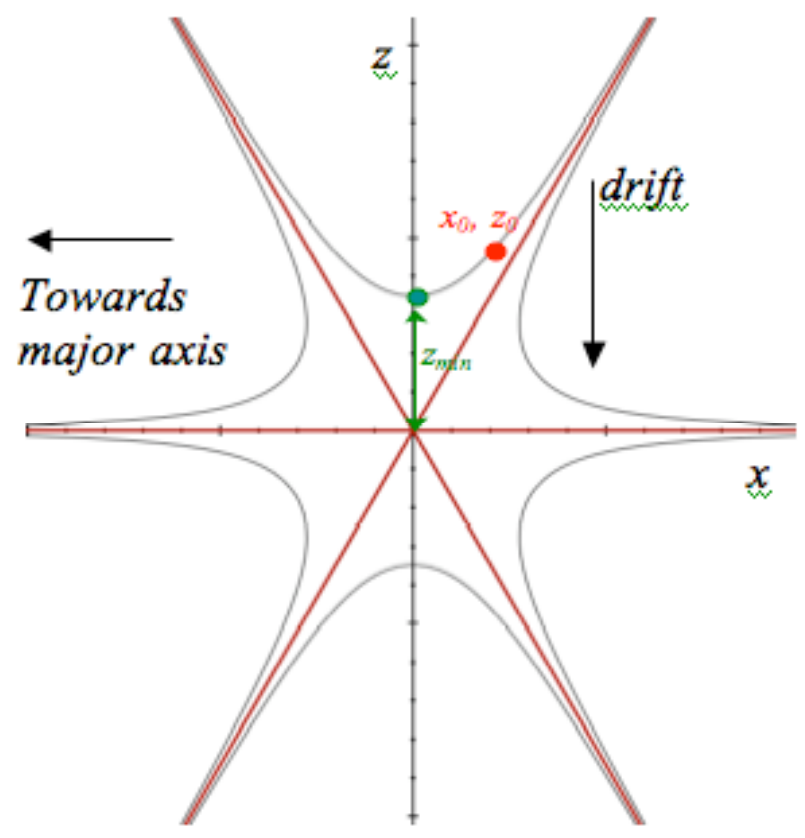

Fig. 1 The geometry of the problem. The separatrix is shown in red; the confinement region is situated between the two upper branches of the separatrix. Shown in black is one of the flux surfaces; $z_{\text {min }}$ marks the flux surface where the particle was initially situated. Note that $\mathrm{Z}_{0}=\mathrm{Z}_{\min }$ only for $\mathrm{x}_{0}=0$. 

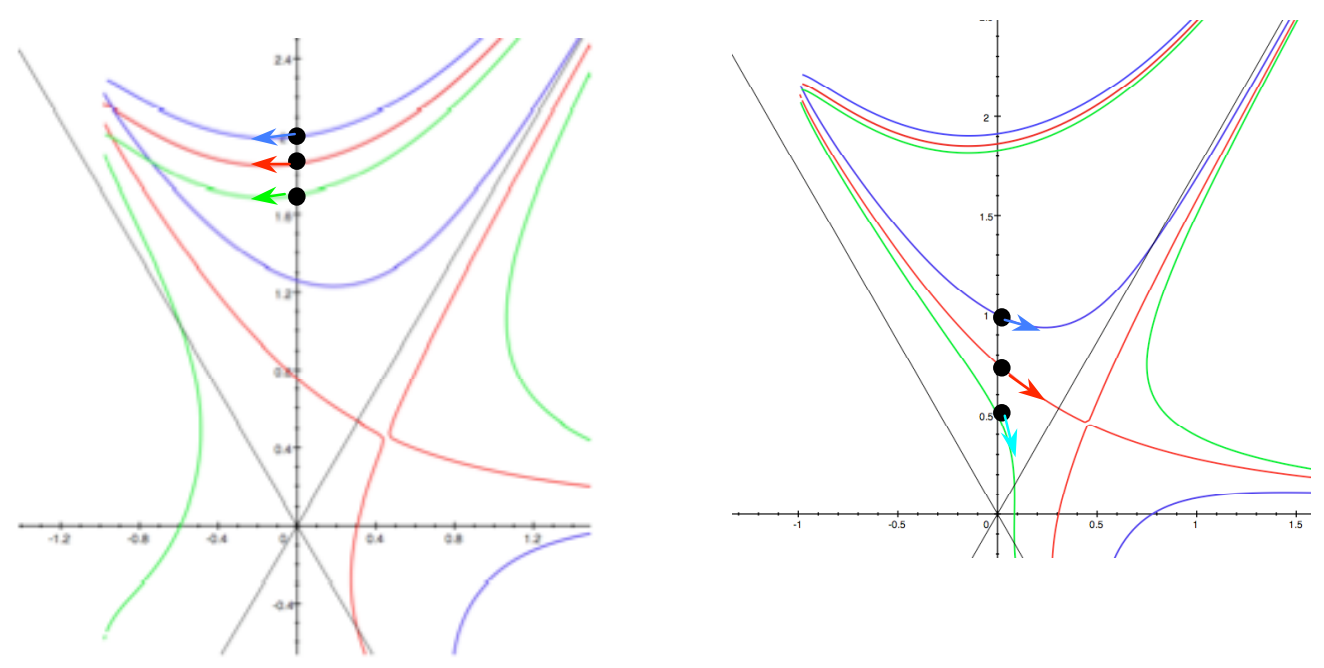

Fig. 2. Particle trajectories in the vicinity of the poloidal field null for the particles starting at $\xi=0$. Black dots are the starting points; arrows indicate initial direction. a) Particles moving to the left (to the high-field side) in the starting point for $\xi *=-$ 1. The red line is a singular self-intersecting trajectory which separates the particles experiencing and not experiencing prompt losses. Note that the losses occur predominantly through the high-field side, with only a relatively narrow region between the null and the loss boundary on the low-field side. b) Particles moving to the right in the starting point. These trajectories are populated by the particles that are reflected in the turning point and not promptly lost. In particular, the green trajectory is not populated. 


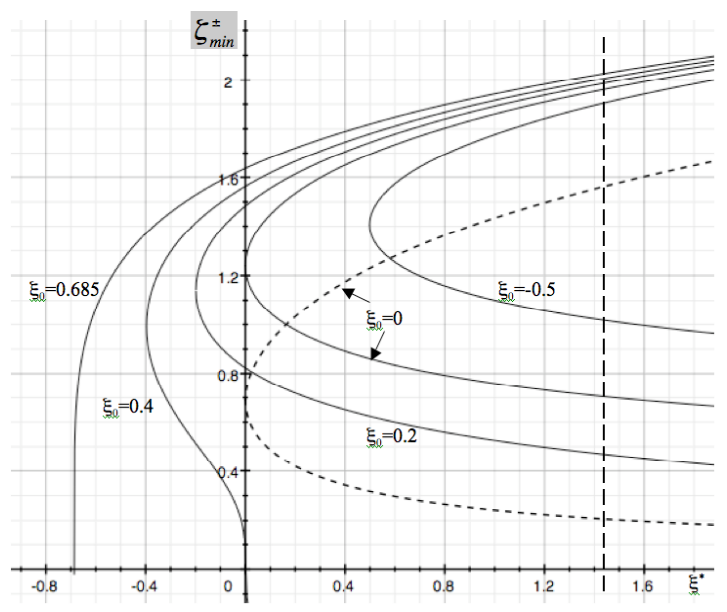

Fig. 3 Dependence of the position of the marginal flux surface on the position of the turning point $-\xi^{*}$ for several values of $\xi_{0}$. The upper (lower) branch is marked in the text by $\zeta_{0 m}^{ \pm}$. For $\xi_{0}>0.685$ only the upper branch remains. For the given spatial point, prompt losses occur for the particles lying below the upper branch for the particles moving to the left, and below the lower branch for the particles moving to the right. Dashed curve correspond to the X-point divertor (Eq. () with $\xi_{0}=0$ ). The vertical dashed line corresponds to $\xi_{\text {crit }}^{*}$ for $a=0.4 \mathrm{R}, \varepsilon=0.05$. 


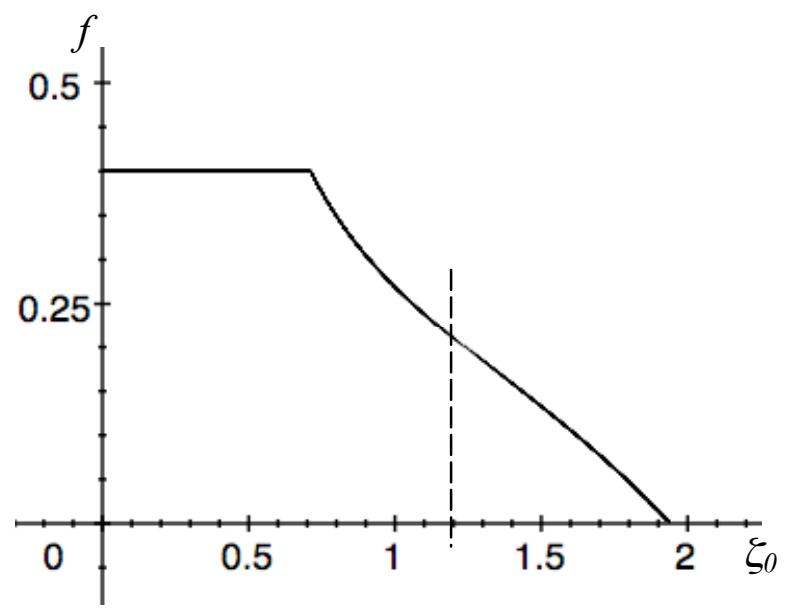

Fig. 4. The dependence of the fraction of the empty part of the phase space on the distance of the starting point from the separatrix, for the particles with $\xi_{0}=0$. Dashed vertical line shows the inflection point (Eq. (11)). 


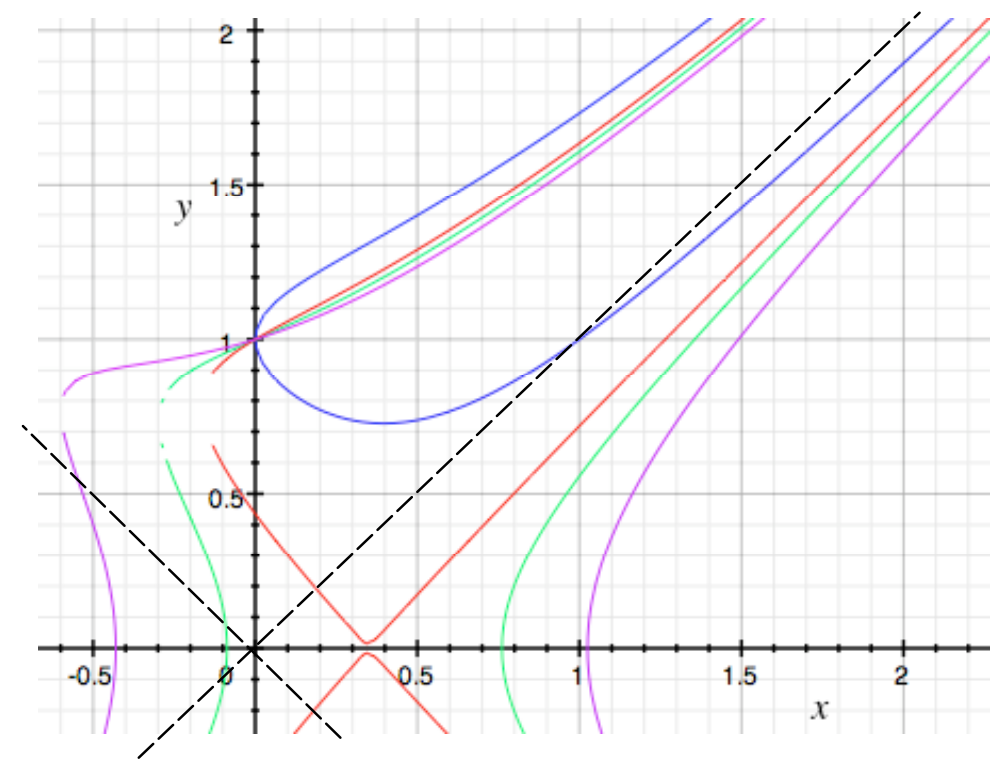

Fig. 5 Particle trajectories in the X-point geometry. The starting point is $\xi_{0}=0, \zeta_{0}=1$. Dashed lines are the branches of the separatrix. 